\title{
Tracing multiple breath markers with flame-made electronic noses
}

\author{
Andreas T. Güntner ${ }^{1}$, Nicolay J. Pineau ${ }^{1}$, Sotiris E. Pratsinis ${ }^{1}$ \\ ${ }^{1}$ Particle Technology Laboratory, ETH Zurich, Sonneggstrasse 3, 8006 Zürich, Switzerland \\ pineaun@ptl.mavt.ethz.ch
}

\begin{abstract}
:
A portable and simple-in-use electronic nose is presented that simultaneously monitors acetone, ammonia and formaldehyde. These compounds are potential breath tracers for fat burn monitoring, kidney failure and lung cancer detection. The array consists of four differently selective and highly sensitive nanostructured metal oxides. Sensing particles are produced by flame spray pyrolysis (FSP) and directly deposited onto silicon wafer-bases microsubstrates with interdigitated electrodes forming highly porous films. This multifunctional microsensor array was tested on simulated breath mixtures with the target tracers at realistic $90 \%$ relative humidity. By applying statistical analysis to the sensor responses, an accurate quantification of the target analytes was achieved. As a result, a compact electronic nose is presented that could be applied in portable breath analyzers.
\end{abstract}

Key words: Breath analysis, sensor array, ammonia, acetone, formaldehyde

An emerging field in gas sensor research is breath analysis, a rapid and non-invasive method for screening and monitoring of diseases. Especially promising as breath marker is ammonia since its concentration is significantly elevated in kidney failure patients (820 - 14'700 ppb) compared to healthy people (400 - 1'800 ppb).[1] Also Acetone, a byproduct of lipolysis, is interesting to monitor as indication of the fat burn intensity during workout in gyms or to guide dieting.[2] Finally, formaldehyde (FA) is a potential marker for lung cancer [3] and could be used for rapid screening.

Required are portable and easy-in-use breath analyser for personal use in a widespread population. Electronic noses are rather suitable as they can analyze several compounds in complex gas mixtures simultaneously. In principle, such an E-nose consists of sensors with different selectivities and their responses are evaluated statistically for combinatorial selectivity, mimicking the human olfactory system.[4] Especially promising are solid-state sensors made of chemoresistive metal-oxides due to their high sensitivities to oxidizing and reducing gases when nanostructured. Such an E-nose has been applied just recently to detect FA selectively down to $3 \mathrm{ppb}$ in simulated breath mixtures.[5]

Flame spray pyrolysis is a great tool to design E-noses. By featuring rapid processing, great material flexibility and superior control over particle size and film morphology, furthermore mixed oxides, solid solutions and metastable phases can be explored with widely tuneable sensing characteristics. This has led to even analyte-selective sensors, as demonstrated with $\mathrm{Si}$-doped $\mathrm{MoO}_{3}$ (ammonia) [6], Ti-doped $\mathrm{ZnO}$ (isoprene) [7]

Here, we demonstrate the accurate ppb-level detection of acetone, FA and ammonia in simulated breath mixtures with an E-nose (Fig. 1 ). It consists of four flame-made and differently doped $\mathrm{SnO}_{2}$ microsensors. Selectivity is obtained by subsequent statistical response analysis by multivariate linear regression.

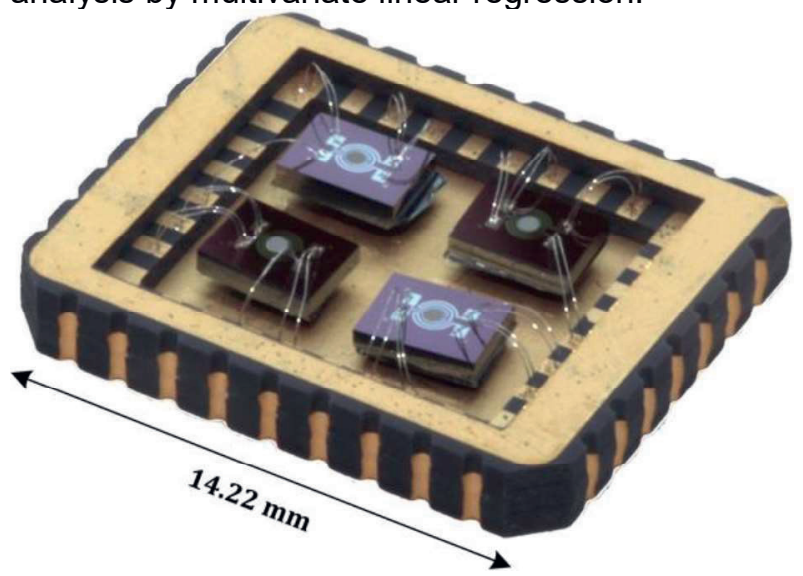

Fig. 1. E-nose consisting of 4 microsensors wirebonded on a chip carrier. Each sensor features a different functional sensing material, namely Pt-, Si-, $P d-$, and Ti-doped $\mathrm{SnO}_{2}[5]$.

In total 60 random 3-analyte combinations of breath-relevant FA $(30,60,90,120,150$ or 180 
ppb), $\mathrm{NH}_{3}(250,500,800,1200,1600$ or 2000 $\mathrm{ppb})$ and acetone $(250,400,600,800,1200$ or $1800 \mathrm{ppb}$ ) were tested. Whereas 20 of the 60 combinations were used for calibration and the remaining 40 were used to assess the E-nose performance displayed in Fig 2.
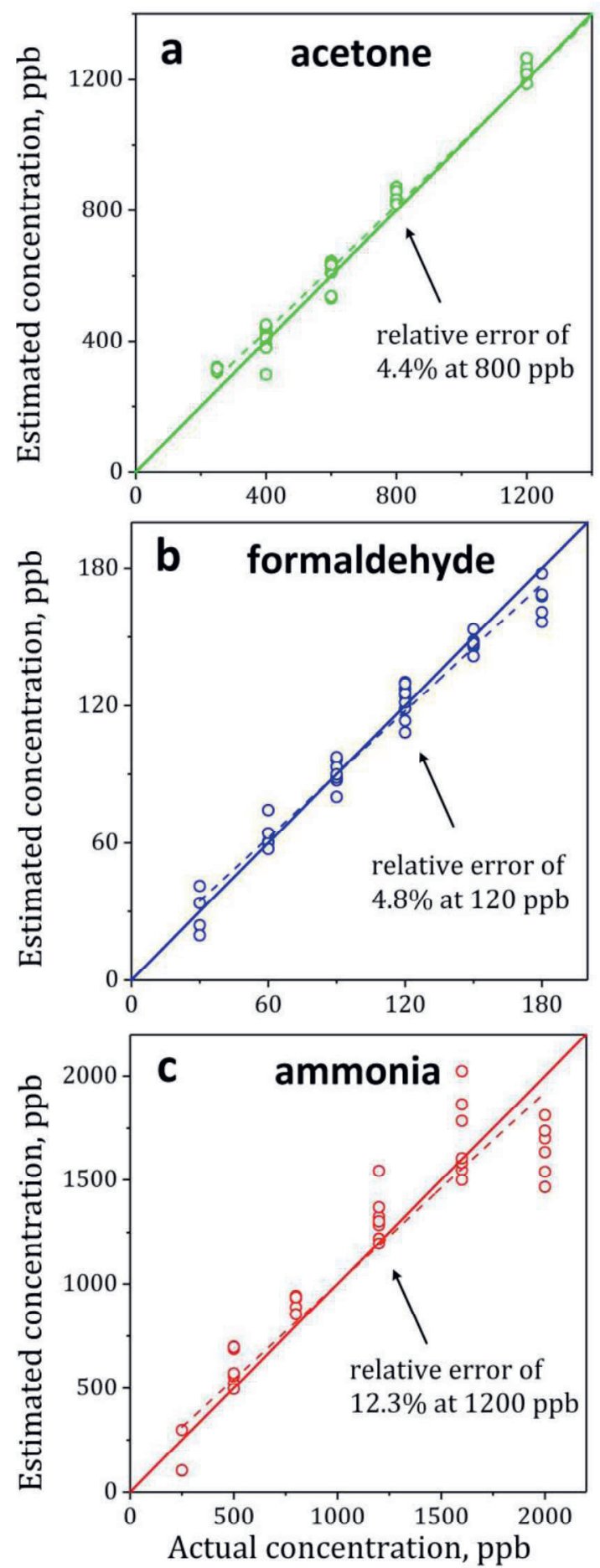

Fig. 2. E-nose estimation of acetone (a), formaldehyde (b) and ammonia (c) in 3-analyte mixtures containing acetone (250-1800 ppb), FA (30$180 \mathrm{ppb})$ and ammonia (250-2000 ppb) at 90\% RH. Model calibration was performed with 20 out of the 60 gas mixtures. Linear fits of the estimated concentrations are indicated as dashed lines.
In fact, when evaluating the E-nose performance in simulated breath mixtures consisting of acetone, FA and ammonia at breath relevant concentrations at $90 \% \mathrm{RH}$, these analytes are estimated with sufficient accuracy. In specific, acetone (Fig. 2a) is clearly resolved over the entire range with low relative error of $4.5 \%$ at $800 \mathrm{ppb}$. Other gases are estimated similarly well with relative errors for FA (Fig. 2b) and ammonia (Fig. 2c) of $4.4 \%$ (at $120 \mathrm{ppb}$ ) and $12.3 \%$ (at $1200 \mathrm{ppb}$ ), respectively. As a result, an E-nose has been developed for simultaneous and accurate detection of acetone, FA and ammonia at $90 \%$ $\mathrm{RH}$ simulated real breath mixtures. These are potential breath tracers for fat burn monitoring, kidney failure and lung cancer detection, respectively. Since this compact E-nose can be installed into a hand-held breath analyzers, it is promising as non-invasive medical device for widespread application.

\section{References}

[1] Davies, S.; Spanel, P.; Smith, D., Quantitative analysis of ammonia on the breath of patients in end-stage renal failure. Kidney Int. 1, 223228 (1997); 10.1038/ki.1997.324.

[2] Güntner, A. T.; Sievi, N. A.; Theodore, S. J.; Gulich, T.; Kohler, M.; Pratsinis, S. E., Noninvasive Body Fat Burn Monitoring from Exhaled Acetone with Si-doped $\mathrm{WO}_{3}$-sensing Nanoparticles. Anal Chem 19, 10578-10584 (2017); 10.1021/acs.analchem.7b02843.

[3] Hakim, M.; Broza, Y. Y.; Barash, O.; Peled, N.; Phillips, M.; Amann, A.; Haick, H., Volatile organic compounds of lung cancer and possible biochemical pathways. Chem. Rev. 11, 5949-5966 (2012); 10.1021/cr300174a.

[4] Persaud, K.; Dodd, G., Analysis of discrimination mechanisms in the mammalian olfactory system using a model nose. Nature 5881, 352-355 (1982); 10.1038/299352a0.

[5] Güntner, A. T.; Koren, V.; Chikkadi, K.; Righettoni, M.; Pratsinis, S. E., E-Nose Sensing of Low-ppb Formaldehyde in Gas Mixtures at High Relative Humidity for Breath Screening of Lung Cancer? ACS Sensors 5, 528-535 (2016); 10.1021/acssensors.6b00008.

[6] Güntner, A. T.; Righettoni, M.; Pratsinis, S. E., Selective sensing of $\mathrm{NH}_{3}$ by $\mathrm{Si}$-doped $\mathrm{\alpha}-\mathrm{MoO}_{3}$ for breath analysis. Sens. Actuators B, 266273 (2016); 10.1016/j.snb.2015.09.094.

[7] Güntner, A. T.; Pineau, N. J.; Chie, D.; Krumeich, F.; Pratsinis, S. E., Selective sensing of isoprene by Ti-doped $\mathrm{ZnO}$ for breath diagnostics. J. Mater. Chem. B 32, 5358-5366 (2016); 10.1039/C6TB01335J. 